\title{
Variant profiling of evolving prokaryotic populations
}

\author{
Markus Zojer ${ }^{1}$, Lisa N Schuster ${ }^{2}$, Frederik Schulz ${ }^{3}$, Alexander Pfundner ${ }^{1}$, Matthias Horn ${ }^{2}$, Thomas Rattei \\ Corresp. 1 \\ ${ }^{1}$ Department of Microbiology and Ecosystems Science, Division of Computational Systems Biology, University of Vienna, Vienna, Austria \\ 3 DOE Joint Genome Institute, Lawrence Berkeley National Lab, Walnut Creek, California, United States \\ Corresponding Author: Thomas Rattei \\ Email address: thomas.rattei@univie.ac.at
}

Genomic heterogeneity of bacterial species is observed and studied in experimental evolution experiments, clinical diagnostics and occurs as micro-diversity of natural habitats. The challenge for genome research is to accurately capture this heterogeneity with the currently used short sequencing reads. Recent advances in NGS technologies improved the speed and coverage and thus allowed for deep sequencing of bacterial populations. This facilitates the quantitative assessment of genomic heterogeneity, including low frequency alleles or haplotypes. However, false positive variant predictions due to sequencing errors and mapping artifacts of short reads need to be prevented. We therefore created VarCap, a workflow for the reliable prediction of different types of variants even at low frequencies. In order to predict SNPs, InDels and structural variations, we evaluated the sensitivity and accuracy of different software tools using synthetic read data. The results suggested that the best sensitivity could be reached by a union of different tools, however at the price of increased false positives. We identified possible reasons for false predictions and used this knowledge to improve the accuracy by postfiltering the predicted variants according to properties such as frequency, coverage, genomic environment/localization and co-localization with other variants. We observed that best precision was archived by using an intersection of at least 2 tools per variant. This resulted in the reliable prediction of variants above a minimum relative abundance of $2 \%$. VarCap is designed for being routinely used within experimental evolution experiments or for clinical diagnostics. The detected variants are reported as frequencies within a vcf file and as a graphical overview of the distribution of the different variant/allele/haplotype frequencies. The source code of VarCap is available at https://github.com/ma2o/VarCap. In order to provide this workflow to a broad community, we implemeted VarCap on a Galaxy webserver, which is accessible at http://galaxy.csb.univie.ac.at. 


\section{Variant profiling of evolving prokaryotic populations}

4

5 Markus Zojer ${ }^{1,2, *}$, Lisa N. Schuster ${ }^{2}$, Frederik Schulz ${ }^{3}$, Alexander Pfundner ${ }^{1}$, 6 Matthias Horn ${ }^{2}$ and Thomas Rattei ${ }^{1}$

7

8 'Department of Microbiology and Ecosystems Science, Division of Computational Systems

9 Biology, Althanstrasse 14, 1090 Wien

10 2Department of Microbiology and Ecosystems Science, Division of Microbial Ecology,

11 Althanstrasse 14, 1090 Wien

$12{ }^{3}$ DOE Joint Genome Institute, 2800 Mitchell Dr, Walnut Creek, CA 94598 


\section{ABSTRACT}

14

15 16 17

Genomic heterogeneity of bacterial species is observed and studied in experimental evolution experiments, clinical diagnostics and occurs as micro-diversity of natural habitats. The challenge for genome research is to accurately capture this heterogeneity with the currently used short sequencing reads. Recent advances in NGS technologies improved the speed and coverage and thus allowed for deep sequencing of bacterial populations. This facilitates the quantitative assessment of genomic heterogeneity, including low frequency alleles or haplotypes. However, false positive variant predictions due to sequencing errors and mapping artifacts of short reads need to be prevented.

We therefore created VarCap, a workflow for the reliable prediction of different types of variants even at low frequencies. In order to predict SNPs, InDels and structural variations, we evaluated the sensitivity and accuracy of different software tools using synthetic read data. The results suggested that the best sensitivity could be reached by a union of different tools, however at the price of increased false positives. We identified possible reasons for false predictions and used this knowledge to improve the accuracy by post-filtering the predicted variants according to properties such as frequency, coverage, genomic environment/localization and co-localization with other variants. We observed that best precision was archived by using an intersection of at least 2 tools per variant. This resulted in the reliable prediction of variants above a minimum relative abundance of $2 \%$.

VarCap is designed for being routinely used within experimental evolution experiments or for clinical diagnostics. The detected variants are reported as frequencies within a vcf file and as a graphical overview of the distribution of the different variant/allele/haplotype frequencies. The source code of VarCap is available at https://github.com/ma2o/VarCap. In order to provide this workflow to a broad community, we implemeted VarCap on a Galaxy webserver, which is accessible at http://galaxy.csb.univie.ac.at. 


\section{INTRODUCTION}

40

41 The genotyping of heterogeneous populations of one prokaryotic species is an increasingly

42 important method to address microbiological questions regarding population composition and

43 dynamics under prevalent selective pressures. This approach is e.g. used in experimental 44 evolution (EE) experiments (Barrick \& Lenski, 2013) and studies of host - pathogen systems 45 (Gardy et al., 2011, Bos et al., 2011, McElroy, Thomas \& Luciani, 2014). Recent developments in 46 Next-Generation-Sequencing (NGS) technologies allow for sequencing at high coverage within a 47 short timeframe, however limited to short read length.

48 The classical approach of assembling genomes out of short DNA reads preferably reconstructs 49 the most abundant genotype into genome contigs and scaffolds. In order to retrieve haplotype 50 frequency information, reads need to be mapped onto the assembly or a reference genome. 51 Variant calling is then performed on the alignment of the reads. The predicted variants can be phased into haplotypes or alleles if a whole haplotype reconstruction is not possible due to insufficient linkage of the variant sites. The variant prediction, however, can lead to false positives due to sequencing errors, such as InDels and substitutions. The reads may be misplaced during mapping due to their short length and thus can lead to false positive variant calls ( $\mathrm{Li}, 2014$ ). Sequencing errors can be partially reduced by quality filtering and error correction (Yang, Chockalingam \& Aluru, 2013). As a consequence, the substitution error rate for Illumina could be decreased below one percent while InDel homopolymer errors showed to accumulate logarithmically with the length of the stretches (Minoche, Dohm \& Himmelbauer, 2011) and can thereby reliably identified.

In evolving populations, we expect a heterogeneous mix of variant alleles (Fig. 1). Most of the genotyping studies of prokaryotes so far have been done by resequencing of clonal bacterial cultures (Maharjan et al., 2013, Blount et al., 2012. The technique of deep sequencing of nonclonal populations, named pool-seq, was mainly done for metagenomic profiling of communities (Qin et al., 2010) and only to a minor extend for the characterization of allele frequencies (Eyre et al., 2013, Khan et al., 2011, Köser et al., 2012, Pulido-Tamayo et al., 2015). The genotyping of non-clonal variants in heterogeneous populations, however, remains challenging (DePristo et al., 2011, Nielsen et al., 2011, Kofler \& Schlötterer, 2014, Pulido-Tamayo et al., 2015).

In order to get a most complete picture of the different haplotype or allele frequencies it is fundamental to use pool-seq and exploit high coverage sequencing data to detect all types of variants, which are SNPs, InDels and structural variations (SV). One way to deal with this is to

72 integrate several variant calling software tools, which utilize different approaches for the detection 
73 of the different kinds of variants.

74 Commonly used tools to identify SNPs are Samtools/bcftools and GATK (Li et al., 2009, McKenna 75 et al., 2010). These tools were developed with the assumption to detect variants within diploid 76 organisms, which limits their detection power for haploid prokaryotes. Therefore, we also 77 considered the more generic tool VarScan2 (Koboldt et al., 2012), which can predict SNP 78 frequencies in low and high coverage data and some specialized tools for variant prediction within 79 high coverage data, such as LoFreq-Star (Wilm et al., 2012), Breseq (Barrick et al., 2014) and 80 FreeBayes (Garrison \& Marth, 2012). Here we used Lofreq-Star, as a previously published 81 evaluation showed it to be superior to Breseq in terms of sensitivity (Wilm et al., 2012). We also 82 evaluated Freebayes, which is widely used in pool-seq experiments for eukaryotes with known 83 pool size but can also analyze a bacterial population with unknown pool size. The tools all work 84 on read alignments or mpileup files and use read and mapping quality scores as well as strand 85 bias filters to reliably detect SNPs. In addition, Samtools/bcftools and VarScan2 and FreeBayes 86 can also be used to identify small InDels. Pindel (Ye et al., 2009) uses a pattern growth algorithm 87 to detect small and large InDels from $1 \mathrm{bp}$ up to 10kb. Large InDels and structural variations (SV), 88 such as translocations, duplications and inversions, are detected by Breakdancer and Delly (Chen 89 et al., 2009a, Rausch et al., 2012), as they make use of insert size deviations, paired end 90 information and split read information to find variations larger than $300 \mathrm{bp}$. As an alternative, 91 Cortex_var (Iqbal et al., 2012) does not rely on mapped reads but uses de novo assembled 92 contigs, which are compared to each other or to a reference in order to identify most kinds of 93 94 variants. All those approaches have been designed for different degrees of zygosity, ranging from diploid genomes to multiploid populations with low abundant genotypes. The genotyping of prokaryote populations in experimental evolution experiments is typically based on many NGS datasets with high coverage. There is therefore a demand for fully automated software for read mapping and variant calling, which is both sensitive and accurate, aware of low abundant subpopulations, and which considers all possible types of variants. To the best of our knowledge no such software workflow has been published so far. In this study, we have evaluated variant callers on synthetic data in order to determine and compare their sensitivity and accuracy. This allowed us to develop and validate VarCap, a workflow for accurate and sensitive genotyping 102 of prokaryotic populations. We finally applied VarCap to a long-term experimental evolution 103 experiment of a bacterial symbiont of amoebae. 


\section{METHODS}

105 Creating synthetic variant genomes: Ideally, the organism selected for simulation should exhibit 106 generic properties that make the results applicable for most prokaryotes. In our simulation and 107 evaluation of the variant detection prototype, however, we decided to pick the non-model 108 organism Protochlamydia amoebophila. It offered the unique opportunity to experimentally 109 validate variant predictions immediately during the software development. In addition, $P$. 110 amoebophila exhibits typical properties as its genome size of $2.4 \mathrm{Mb}$ is close to one of the main 111 peaks in the bacterial and archaeal genome size distribution (Koonin \& Wolf, 2008). For validation 112 purposes, we additionally used 6 different organisms that we selected to represent the diversity 113 of prokaryotic genomes regarding $\mathrm{G}+\mathrm{C}$ contents and genome size.

114 Variant datasets were created by randomly inserting different types of variants into reference 115 genomes downloaded from the NCBI Refseq database (Pruitt et al., 2012) (Suppl. Table 1). We used a SNP/InDel ratio of 10 for small InDels and 20 for all InDels, as SNP/InDel ratios for bacterial genomes were often reported between 15 and 20 (Moran, McLaughlin \& Sorek, 2009, the process of horizontal gene transfer. As structural variations are reported to be crucial for bacterial genome evolution, we also added few translocation, duplication and inversion sites to challenge the detection software.

We created mixed types of datasets containing 135 variations, as well as datasets containing one specific type of variant. The 135 variants of the mixed type dataset consisted of 100 SNPs, 10 small InDels, 10 large InDels and 5 translocations, 5 duplications (including one double duplication) and 5 inversions (Set: sim_135VAR, Suppl. Table 1).

127 positioned as single seeds, to which the other SNPs were randomly assigned with decreasing 128 probability. The maximum number of SNPs within a hotspot was 4 , which were randomly placed within a distance of 4 to 60 bases. The size of the large InDels was randomly chosen between 5 and 2000 nucleotides, while the size of translocations, duplications and inversions varied from 300 to 2000 nucleotides. The datasets harboring only one type of variant contained either 100 SNPs, 100 small InDels, 100 large InDels, 50 translocations, 50 duplications or 50 inversions (Sets sim_100SNP, sim_100IndS, sim_100IndL, sim_50ITX,sim_50DUP,sim_50INV).

ALFSim is a genome evolution simulator and was used (Dalquen et al., 2012) to simulate the evolution of more distantly evolved subpopulations. Therefore, coding and intergenic nucleotide sequences according to the genome annotation were extracted from the genome reference fasta file. This extracted sequences served as input for ALFSim. From the ALFsim output, we selected 
138 a simulated subspecies having a nucleotide dissimilarity of $0.8 \%$ resulting in 21000 SNPs, 100

139 InDels and 3 duplications. The resulting fasta file was used for read simulation, construction of a 140 heterogeneous population and prediction of variants.

141

142 Sequencing read simulation: We used SimSeq (https://github.com/jstjohn/SimSeq, version from 143 4.12.2011, (Earl et al., 2011) and pIRS (Hu et al., 2012) for the simulation of 100 nucleotides (nt) 144 paired end Illumina reads. The reads were simulated with an insert size of $250 \mathrm{nt}$ and an insert 145 size standard deviation of 10,20 and $30 \%$. For pIRS we used the supplied error model, while for 146 Simseq the updated empirical error models for forward and reverse strand were used 147 (hiseq_mito_default_bwa_mapping_mq10_1_Corrected.txt, hiseq_mito_default 148 _bwa_mapping_mq10_2_Corrected.txt). We simulated minor allele frequencies (MAF) by mixing 149 simulated reads from the original reference with simulated reads from the variant datasets. 150 Thereby we created MAF of $40,20,10$ and 4 percent.

151

152 Sequence read processing and mapping: The quality of the simulated reads was determined 153 using FastQC (v0.10.0, (Patel \& Jain, 2012). The quality filtering and trimming of the simulated 154 and the sequenced reads was done by Prinseq-lite (0.19.5, Schmieder \& Edwards, 2011 and 155 Trimmomatic (0.32, Bolger, Lohse \& Usadel, 2014) and applied with the following settings: First 156 a sliding window with size 10 removed any bases with lower quality than 20 starting from the 3' 157 side by cutting off the read part containing the low-quality bases. The sliding window approach 158 159 160 161 162 163 164 165 166 167 168 has the advantage that low quality bases are also removed within the read and not only at the end (which is done, if read trimming is done only from the 3' of 5' end). We removed all reads shorter than $40 \mathrm{nt}$. To remove low quality reads, we discarded any read with an average Phred score below 30. Only read pairs were kept. These reads were mapped against the reference genome using bwa-mem (bwa-0.7.5a, (Li, 2013) (Li \& Durbin, 2009) with standard settings and stored as bam files. For conversions from sam to bam files and from bam to fastq files (as Cortex_var input) we used samtools (0.1.18, Li et al., 2009) and picard-tools (v1.92, http://picard.sourceforge.net/).

Mapping artifacts: In order to emulate mismapped reads due to an incomplete reference genome, we mapped reads that were generated from an updated (newly assembled) reference genome back to the older and about $20 \mathrm{kB}$ shorter version and to the current version. This dataset did not 169 contain any simulated variants. 
171 Variant calling: In order to assess true and false positive variant detection rates, artificial non172 clonal populations containing SNPs, InDels and SV at abundances of $40 \%, 20 \%, 10 \% 5 \%$ and $1732 \%$ were simulated. We used samtools/bcftools (0.1.18, Li et al., 2009), GATK-lite 174 (GenomeAnalysisTKLite-2.2-8, McKenna et al., 2010), VarScan2 (2.3.6, Koboldt et al., 2012, 175 LoFreq (0.6.1, Wilm et al., 2012 and LoFreq2 (lofreq-star 2.0 .0 beta 1, unpublished). For the 176 detection of small InDels we used VarScan2 and pindel (024t, Ye et al., 2009). For large InDels 177 and structural variations (SV) we used pindel, which is described to work well between on 178 variations between 1 and 1000 nt, breakdancer (breakdancer-1.1_2011_02_21, Chen et al., 179 2009a and delly (0.0.11, Rausch et al., 2012) (both start calling SV at $300 \mathrm{nt}$ ). Additionally, we 180 used the assembler cortex_var (CORTEX_release_v1.0.5.14, Iqbal et al., 2012), which can detect 181 variations by comparing assembled contigs to a reference genome sequence. The sensitivity and 182 precision of the combined workflow were calculated as: sensitivity $=$ TP / (TP+FN), and precision $183=\mathrm{TP} /(\mathrm{TP}+\mathrm{FP})$. The TP, FP and FN are measured per variant, giving e.g. a SNP and a large 184 deletion event the same weight.

Setting the minimum abundance for a variant: In order to call a variant, it has to be present within 187 a minimum count of sequencing reads. Some variant callers need a variant to be present on 4-8 reads, so we set 8 reads as the minimum absolute abundance (MAA). However, as read coverage slightly varies along the genome, we also used minimum relative abundance (MRA), which is the percentage of variant reads compared to the total coverage. So, a MAA of 8 reads corresponds to a MRA of $2 \%$ at $400 x$ total coverage.

192

Examining the similarity of repetitive regions: We used the edit distance in order to measure the 194 similarity of repetitive regions. The edit distance measures the similarity of 2 sequences by counting the differences between them. This difference can be a substitution, insertion or deletion of a nucleotide. Therefore, an edit distance of one means that two sequences differ in either a substitution, insertion or deletion of a nucleotide.

Analysis of a long-term experimental evolution experiment: We applied the VarCap workflow to a long-term experimental evolution experiment in order to evaluate its performance on Illumina PE data. Two independent laboratory cultures of the amoeba symbiont Protochlamydia amoebophila were subjected to NGS sequencing using the Illumina Genome Analyzer II platform (100bp PE reads, 250bp insert size, 3000x coverage, 250bp insert size) about nine years after its genome was initially sequenced by Sanger sequencing (Horn et al., 2004) (Available at the SRA: 
205 SRR5123091). For analysis, the obtained Illumina reads were randomly split into replicate read 206 packages with 250-fold coverage each and utilized to detect variant sub-populations at different 207 abundances.

208

PCR verification of variations: To verify the variations at positions 1339224,1339720 , and 2101338568 in the genome of $P$. amoebophila we amplified the region 1338371-1339843 by PCR 211 using the primers LS0003 5'-AGCTGCATCATTTATCTTCTAG-3' and LS0004 5'212 ATCAGTCCACCTACTATCATG-3'. The obtained 1472 bp fragment was cloned into the pCR4213 TOPO vector (Invitrogen). Subsequently, 16 of the obtained colonies were picked, and the 214 presence of variations in the cloned amplicons was checked. Clones were sequenced by Sanger 215 sequencing with the primers T3 and T7. Similarly, 14 putative variations in a repetitive region 216 between positions 1533689 and 1534636 were assessed using the primer pair LS0005 5'217 TCTCTAGCTCTTTCGCAAATTG-3' and LS0006 5'-CAGTGTTTAACTGGCTGAAAC-3'.

219 A Galaxy instance of VarCap

220 We simplified the use of VarCap for non-experts to a 3-step process facilitated by our Galaxy 221 server (Afgan et al., 2016): I) Create account and login, II) Upload your data to Galaxy and III) 222 Run the VarCap workflow. After the workflow is finished, the user is informed via Email notification. 223 The results are viewable at and downloadable from the website. The output files consist of a vcf 224 file with a detailed description of the variants as wells as two pdf files, which contain overview 225 information about variant and total coverage and frequency information. 


\section{RESULTS}

227

228 Determination of methods capable of sensitive detection of low abundant variations

229

230 Evaluation strategy

231

232 At the moment, there is no software tool or method, that could detect all different types of variants 233 simultaneously, which are relevant for prokaryotic genomes. Therefore, we separately evaluated 234 variant detection tools for SNPs, InDels and structural variants (SV). Representative methods for 235 these three targets were selected according to their underlying methodologies. In order to identify 236 the variant calling tools that most sensitively and reliably detect low abundant variant, we initially 237 utilized our most basic variation model (sim_135VAR). It incorporates examples of the typical and 238 expected types of variations in microbial genomes, located in typical distances to each other. 239 From these results, we constructed a preliminary software framework, which was used as basis 240 for the further evaluations and improvements.

242 SNPs

Among the many available SNP calling software tools we have compared LoFreq-Star, Varscan2, GATK, Samtools/bcftools, Freebayes and Cortex_var. All of these tools, except Cortex_var, rely on the mapping of reads to a known reference. Cortex_var, instead, de novo assembles variant reads into contigs and thereby detects SNPs. Samtools/bcftools and GATK were only designed for homozygous and heterozygous genomes (Yost et al., 2013), whereas LoFreq-Star, Varscan2 and Cortex_var should be able to detect low frequency variants from high coverage sequencing data. Variants were simulated at minor allele frequencies (MAF) of $40 \%, 20 \%, 10 \%$ and $4 \%$ and evaluated at minimum relative abundance (MRA) cutoffs of $20 \%, 10 \%, 5 \%$ and $2 \%$ accordingly.

252 This means that ideally all variants present at and above those frequencies should be detected. At MRAs of $20 \%$ and $10 \%$, variants were detected by all SNP calling software tools at a similar sensitivity (Fig. 2 A). According to the expectations, the detection rate of GATK and Samtools/bcftools was worse compared to the other programs when the MRA was reduced to $5 \%, 2 \%$ and $1 \%$ (Fig. 2 A). At a low MRA of $1 \%$ LoFreq-Star shows less sensitivity than Varscan 2. This is to be expected, as LoFreq-Star generates its own detection threshold based of coverage and quality to avoid FP and therefore detects no variants below that threshold (Fig. 2 A). The price of the higher sensitivity of Varscan2 at MRA of $1 \%$, however, comes at the price of elevated 
260 FP variant predictions. Freebayes was able to detect variants at all MRAs with similar sensitivity

261 (Fig. 2 A). However, we observed FP at MRAs of $2 \%$ and $1 \%$ and therefore did not include this 262 tool in further analysis.

263

264 InDels

265

266

Varscan2 and Pindel were used for the detection of small InDels, and Pindel, Breakdancer, Delly and Cortex_var for the detection of larger InDels. For small InDels, the MSA approach used by Varscan2 should perform at a similar rate as the pattern growth algorithm used by Pindel. Pindel, however, is designed to detect InDels from 1-10000bp as it uses a mapping/pattern growth/split read approach. Therefore, it should be able to detect the positions of small and large InDels with base pair precision. Breakdancer and Delly are designed for the detection of InDels larger than $300 \mathrm{bp}$. They use paired end read information for InDel detection, therefore the position of the large InDels may not be reported at bp resolution. Cortex_var is expected to be less sensitive because of the de-novo assembly approach, however it can supply more information than the mapping approaches, including e.g. position, length and sequence of an insertion. The detection rate of InDels showed little effect to different MRA values (Fig. 2 B) (except Samtools/bcftools, see discussion above). Instead, the sensitivity is related to the methodology underlying the software. We observed that Varscan2 can only detect very short InDels (1bp) with the same sensitivity as Pindel, which detected all sizes of InDels with high precision. According to our expectations Breakdancer should have a diminished detection rate for large insertions, as it only considers information about insert size deviation of paired reads and regions with an increased number of anomalous read pairs. We found, that it detects $100 \%$ of all large deletions but misses all insertions. As expected, the assembly method used by Cortex_var performs inferior compared to the others. However, it was one of the only two tools that were able to detect large insertions. It detected one third of the large insertions and reported the inserted sequence, while pindel detected the position of large insertions at a higher rate, but without revealing any details.

For the detection of SV, we used Pindel, Breakdancer and Delly, and we added Cortex_var specifically for inversion detection. These programs differ slightly in their methodological

292 approaches. We expected Delly to be superior to Breakdancer because of the additional split read 293 alignment. Moreover, we expect a limitation of Pindel at larger rearrangements, because the 
294 pattern growth algorithm is used within defined limits (up to 10kb). All tools should be able to 295 detect inversions; however, they are reported as being harder to detect than other SVs. 296 Breakdancer and Delly detected SV, like duplications and transpositions, regardless of the MRA 297 with high sensitivity (>90\%). As expected, the detection rate of Pindel is lagging behind $(80 \%)$ 298 according to of the suggested internal limits of $10 \mathrm{~kb}$. However, the pattern growth method of 299 Pindel was more precise in terms of position and length of the SV as it always hit the exact starting 300 position while Breakdancer and Delly can be off up to 70 bases (Fig. 2 C). We additionally found, 301 that large InDels were called at the sites of translocations events (Fig. 2 C). This is not entirely 302 unexpected, as a translocation consists of an excision and the consecutive insertion of the 303 excised genomic fragment. The excision can also be seen as a deletion of a fragment and is 304 therefore a partial detection of a more complex type of variant.

305 Inversions, however, could only be detected at a minor fraction as break positions by Pindel ( $70 \%$ 306 as break positions) and as inversion by Cortex_var (10\%) (Fig. 2 C inv).

307

308

\section{Selected software tools for VarCap}

309

310

We use LoFreq-Star and Varscan2 for SNPs and Varscan2 and Pindel for small InDels for composing VarCap because they showed similar sensitivity although using different methodological approaches. For larger variants or SV, we observed that a combination of pattern growth, split read and paired end read information approaches, which are used by Pindel, results in high sensitivity. This method works well within defined limits (1-10kb). By using only paired end information (Breakdancer), it is possible to detect larger variants at the cost of a lower length limit (300bp) and a coarser resolution of the variant position. Cortex_var, however, was inferior in sensitivity but revealed more information about the detected variants by using a de-novo approach. This information can be used to correctly identify the type, position, length or sequence of the variant. Therefore, we use Pindel, Breakdancer and Cortex_var for large InDels and Breakdancer, Delly, Pindel and Cortex_var for SV.

321 Due to the different variant calling abilities of the different tools at low frequencies, we combined 322 different tools to increase the sensitivity (Fig. $3 \mathrm{~A}$ ). Beyond sensitivity we also monitored the 323 precision of the different tools for each type of variant in order to avoid methods that have 324 excessive numbers of FP (Suppl. Fig. 1). As a consequence, Cortex_var was used to predict 325 InDels and inversions but not for SNPs as it accumulated many false positive SNPs in certain 326 areas at low frequencies. We also discontinued to use Freebayes for SNP calling, as it showed 327 low precision at MRAs of $2 \%$ and $1 \%$. Taking together all selected software tools we were able to 
328 detect all variants, except inversions, at a MRA of down to $2 \%$ with high sensitivity (Fig. 4). 
329 VarCap - a variant calling workflow with high sensitivity and specificity

330

331 False Positives due to sequencing errors

332

333

False positives occur due to sequencing errors, which are typically present at and below a rate of $1 \%$, therefore we expect them to cause FP calls at and below this relative abundance. In order to study the influence of sequencing errors on different software detection tools, we analyzed 7 differentially composed samples and focus on MRAs of $2 \%$ and $1 \%$ as this seems to be the critical boundary for FP prediction (mono_02-07). At a MRA of $2 \%$ we observed a false positive rate for SNPs, small InDels and Duplications of 0.5 to 1 FP per Megabase (Mb) (Suppl. Fig. 2 B: MRA 2). At a lower MRA of 1\%, we observed an increase in FP (Table 1). At a MRA of 1\%, we could nearly completely find all types of variants, except inversions, which we could identify at a rate of $95 \%$.

341 However, the false positive rate for SNPs increased to 80 FP per Mb, while the FP rate for other 342 variants stayed below one FP per Mb (Suppl. Fig. 2 A, B: MRA 1). This clearly demonstrates that 343 false positive SNPs are caused by sequencing errors, while the other types of variants stayed at 344 the low rate $(\sim 1 \mathrm{FP} / \mathrm{Mb})$.

345 In order to get more insights about the other FP, we examined them in detail at both MRAs. We 346 found that FP of small InDels locate within repetitive regions of the genome. These regions are almost identical areas of the genome at a size that is longer than the insert size of the reads and have an edit distance of 3 or less. Due to their similarity, variant reads can be mapped to similar regions and cause FP calls there. In order to evaluate how MAA and coverage influence the FP rate, we simulated sequencing coverage from 80 to 1600x (using the sim_135VAR dataset) and used MAAs from 4 to 20 to remove FP from the unfiltered variant predictions (Fig. 5). For each coverage/MAA setting. we report the resulting calculated MRA. We report the FP rate as FP per $\mathrm{Mb}$, as with this normalization step we are independent of the genome size. Otherwise a $4 \mathrm{Mb}$ sized genome would produce twice as many FPs as a $2 \mathrm{Mb}$ genome. We detected, that it is necessary to use an MAA cutoff in addition to an MRA cutoff to avoid FP calls at lower coverages (Fig. 5, see FP counts at MRA2 at coverage 160x). 
363 Mismapped reads have been reported as the cause of FP ( $\mathrm{Li}, 2014)$. Thereby incomplete 364 reference genomes lead to reads getting mapped to similar regions and cause FP calls there. To 365 review this finding at a MRA of $2 \%$, we mapped reads without variants back onto an artificially 366 shortened reference genome. We observed 180 FP SNPs/75 FP per Mb which were present at 367 different abundances (20\%, 8\%, 3\%) and grouped into hotspots (Fig. 6 A). False positive variants 368 were not observed when mapping the reads to the correct reference (Fig. 6 B). This finding 369 strongly supports our assumption that wrongly mapped reads cause FP variant calls. A closer 370 investigation of the relevant regions revealed the presence of neighboring break positions, which 371 may indicate both: either a larger structural variation or mismapped reads due to an incomplete 372 reference genome.

373 To identify possible false positives due to mismapped reads, we implemented the following 374 filtering steps: As suggested in prior discussion of this topic ( $\mathrm{Li}, 2014)$ we used the coverage 375 information at the variant sites to tag possible false positives. However, coverage information 376 alone is too coarse for the resolution of low frequent FP. Therefore, we additionally monitor break 377 positions that flank or reside at the variant positions to identify regions with mismapped reads. As 378 all FP were present as small clusters or hotspots, we tagged regions that hosted more than 4 379 SNPs within a sliding window at the double length of the insert size and were accompanied by a 380 break position (BP) as possible FP causing regions. With the application of these filters we could 381 identify and exclude the FP calls (Fig. $6 \mathrm{C}$ ).

382 A closer look at inversions revealed, that they were mostly not identified as inversions but the 383 start and the end point of the inversion were marked as break positions (Suppl. Table 2). Break 384 positions occur because only one read of a pair can be mapped, leading to an accumulation of only forward or reverse reads. They indicate a larger sequence difference between the reads and the reference and are therefore a more general indicator of a larger structural variation. Therefore, these calls represent a partial resolution of the variant. In order to identify and exclude false positives we apply the following filters: To avoid FP SNP calls caused by sequencing errors we apply a MRA of $2 \%$. To avoid FP due to reads mapped to repetitive regions, we mask nearly identical regions according to the properties described above within the reference genome and tag variants that are found within these regions. In order to resolve FP that are caused by incomplete detection of the true variant type, we prioritize larger over smaller variants. Thereby we assign smaller variants to larger ones, if they describe a component of the whole variation e.g. large InDel at excision site of translocation. 
398 We observed that a gain in variant calling sensitivity decreased the precision. Therefore, we 399 added a post-filtering step to the workflow in order to eliminate possible FP. We incorporated a 400 post-processing step for each variant that aims to eliminate FP due to sequencing errors, 401 repetitive regions, partially detected variants and mismapped reads due to reference 402 incompleteness. As a consequence of the dissimilar variant detection rates of some methods, we 403 decided to use more than one tool for each type of variant. In order to gain precision and 404 robustness, for high confidence variants, we required an intersection of predictions per variant. 405 Thereby a variant call had to be supported by at least two different tools. This step further 406 contributed to an improved precision at low MRA cutoffs (1\%), while the detection rate was only 407 slightly diminished (Table 1). This finding is backed up by a recent publication, which made a 408 similar observation regarding the intersection of different tools (Kofler et al., 2016). 
409 Genotyping of diverse synthetic prokaryotic populations

410

411 Detection rates in different genomes

412

413 Genomes exhibit different properties, such as $G+C$ content and size, which could potentially affect 414 the sensitivity and accuracy of variant calling. Therefore, we evaluated our variant calling workflow 415 on six different genomes. These organisms consisted of five bacteria and one archaeon, with 416 differing $\mathrm{G}+\mathrm{C}$ content ranging from 26 to 72 percent as well as a differing genome size ranging 417 from 0.68 to $8.66 \mathrm{Mb}$. The workflow was used with a MRA of $2 \%$ as well as at a MAA of 8 reads 418 supporting a variation. In concordance to our previous results we could detect most of the 419 (simulated) variants (>90\%). However, at a MRA of $2 \%$ we could not observe any dependency 420 on $\mathrm{G}+\mathrm{C}$ content or genome size while the MAA of 8 reads resulted in fewer variant detections at 421 high $\mathrm{G}+\mathrm{C}$ content and genome size (Fig. 7). This observation confirmed our previous observations 422 to use a MRA as a general minimum cutoff for variant detection as it showed little influence to 423 different genome properties. This, however, does not remove the need for a fixed MAA in case of 424 low coverage regions.

425

426 Detection rates in a distantly evolved population

427

428 More distantly evolved populations may lead to a higher number of variants if they are under 429 positive selection. This could affect the sensitivity of variant calling. Therefore, ALFSim (Dalquen 430 et al. 2012) was used to simulate a more distantly evolved population by integrating evolutionary 431 changes (SNPs, InDels and duplications) into the $P$. amoebophila genome. The evolved genome 432 showed a similarity to the reference around $99 \%$, as it contained around 21000 SNPs, 100 InDels 433 and three gene duplications.

434 We evaluated the sensitivity of the variant calling by VarCap at a low abundant subpopulation of $4354 \%$. We used a MRA of $3 \%, 2 \%$ and $1 \%$ as well as a MAA of eight reads (equals a MRA of $2 \%$ in 436 a 400x covered genome). Depending on the minimum abundance requirements, we were able to 437 detect between $90 \%$ and $99 \%$ of all SNPs, between $74 \%$ and $94 \%$ of all InDels and 2 out of 3 438 duplications. The true positive detection rate of SNPs increased to $98 \%$, while the false positive 439 rate remained below $0.3 \%$ when lowering the MRA from 3 to $2 \%$. However, if we lowered MRA 440 further to $1 \%$, we increased the TP rate to $99 \%$ while augmenting the FP rate close to $400 \mathrm{FP} / \mathrm{Mb}$ 441 (Fig. 8A). At a MRA of $2 \%$ we could locate most FP within repetitive regions and recent 442 duplications (Fig. 8B), while at a MRA of $1 \%$ we detected mainly FP caused by the sequencing 
443 error rate (Fig. 8B). At a MRA of $2 \%$, we were able to detect over $90 \%$ of all InDels including all 444 small InDels (size=1), without experiencing false positives (Fig. 8A). With regard to duplications 445 we were able to find two of them at most MRAs, while missing out the shortest one constantly 446 (Fig. 8C SV(DUP)). These findings confirm that we are able to achieve a high accuracy even if 447 the evolved genomes are rather dissimilar. However, a novel finding was that also recent 448 duplications can lead to wrongly placed reads as they are similar to repetitive regions. Therefore, 449 we also included tagging of duplicated regions as possible regions for FP calls into our workflow. 450

451 Detecting variants in a real bacterial population after long term cultivation 452

453 In order to predict variant frequencies within an evolving population, the variant calling workflow 454 was applied to a long-term cultivation experiment of $P$. amoebophila. Different MRA cutoffs from $45520 \%$ to $2 \%$ were used and revealed that variants were present at frequencies down to $2 \%$ (Fig. 456 9A, outer rings). Variants within repetitive regions (Fig. 9A, inner connective lines) were tagged 457 for further inspection. At a MRA of $2 \%$ we observed a total number of 71 variants, which comprised 458 of 34 SNPs, 20 InDels and 17 structural variants. The SNPs and small InDels were annotated 459 using SNPEff (Cingolani et al., 2012). This revealed, that around 83\% of them were situated within 460 coding regions (Suppl. Table 3). At a MRA of $2 \%$ we could find three InDels present at a MAF of $4612 \%$ and one InDel at a MAF of $3 \%$, which were located within homopolymeric regions of length 46210 or longer (Suppl. Table 4). Thus, those InDels were tagged as probable FP for further manual 463 inspection.

464 For the validation of the variant calling prototype of VarCap we picked three variations for further 465 analysis that were present at abundances of $4 \%, 11 \%$ and $28 \%$, accordingly. We performed PCR 466 of the regions surrounding the three variants, cloned the fragments into vectors and picked 16 467 clones of each variant for Sanger sequencing (Table 2, Suppl. Files 1-2). We were able to detect 468 all three variants and thus could confirm the predictions of the VarCap software. 


\section{DISCUSSION}

470

471 Population genomics of microbes is most powerful if we meet the challenge to detect all types of 472 genomic variations even at low frequency. We therefore developed, evaluated and validated 473 VarCap, a workflow that allowed us to reliably identify variants even within low abundant alleles. 474 Increasing sensitivity: We tested the capabilities of the relevant variant calling tools and observed 475 substantial sensitivity differences between the different methods. In order to improve the overall 476 sensitivity, we decided to integrate different tools for variant detection into a combined workflow, 477 in which every variant can be detected by more than one caller.

478 Increasing precision: As more tools are likely to introduce more errors, we also optimized the 479 overall precision. Detecting sequencing errors and mismapped reads was key to control the rate of false positives. When dealing with sequencing errors, we observed that for SNP detection a MRA cutoff of $2 \%$ was sufficient to keep a safety margin to false positives appearing at a MRA cutoff of $1 \%$. Although we found, that a relative abundance cutoff (MRA) is superior to an absolute cutoff (MAA). We also observed that a MRA cutoff leads to FP if the read coverage is too low (<200x). Therefore, we also apply a fixed MAA cutoff of 8 reads to remove FP at low coverage positions. This implies, that for detecting a subpopulation present at a MAF of $>2 \%$ we need a minimum sequencing coverage of 400x. Sequencing experiments should therefore aim for at least $500 x$ to account for reads removed by quality filtering and fluctuations in coverage along the 488 genome.

489 We could not detect any FP InDels within our simulated data but detected several spurious InDels 490 in homopolymer regions of the re-sequencing experiment. These are probably sequencing/PCR 491 artifacts that are not introduced by read simulators. Based on our findings InDels below a MRA of $49210 \%$ should be tagged as potentially false positive if they are located within a homopolymeric 493 region (>8 bases).

494 Mismapped reads can occur within repetitive regions, undetected duplications, or incomplete 495 reference genomes. Therefore, we flag repetitive regions greater than the insert size in order to 496 mark variants appearing within these regions for further inspection. Unnoticed duplications or 497 incomplete references cause reads to get mapped to similar regions, which can be observed by 498 higher coverage and/or variant accumulation within these areas. In order to overcome false 499 positives by misplaced reads, we removed variants that at least fulfill two of the four following 500 rules: I) Either variants lie within regions with a coverage of $20 \%$ above the average and/or II) if 501 there is a break position detected at or within read length of the variant site and/or III) if they lie 502 within a repeat region and/or IV) if more than 5 variants lie within the length of one insert size. 
503 The efficiency for FP removal for each rule may differ among experiments as they dependent on 504 organism, experiment setup, sequencing and reference quality. Therefore, we strongly suggest 505 to use all rules in combination for a most flexible removal of FP predictions due to misplaced 506 reads.

507 Intersection of predictions: We remove FP caused by sequencing errors, Fp due to homopolymer 508 errors and FP due to misplaced reads for all variant calls generated by the different tools. 509 Furthermore, for extracting high confidence variants, we performed an intersection of different 510 tools per predicted variant. Thereby we requested each variant to be confirmed by at least two 511 callers, except for break positions, inversions and large insertions. Inversions and large insertions 512 are harder to detect than other variants. Therefore, an intersection would further decrease their 513 count. Break positions, on the other hand, do not lead to FP predictions. They just indicate 514 problems in mapping, which can be due to structural variants or uncomplete/distant references.

515 Limits of variant detection: We observed that insertions and especially inversions were harder to 516 detect than the rest of the variations. This is not unexpected, as current methods for their 517 prediction need sufficient support by reads, which may get lost at low frequencies. In the simulated 518 evolution data, we missed the shortest duplication constantly. This may be related to a 519 combination of callers working at their operational limits (300 bp) and a diverging evolution of the 520 duplicated sequence due to newly introduced SNPs.

521 According to our results, we could establish rules for filtering out errors and help with the 522 interpretation of different types of variations (e.g. SNP, duplications). Using these rules, we have 523 built a fully automated workflow that reliably predicts rare variants in deep sequencing data.

\section{CONCLUSION}

525

526 We created VarCap, a fully automated workflow that allows scientists to rapidly predict variants 527 within high coverage, short read paired end sequencing data. VarCap automatically performs 528 quality filtering, mapping, variant calling and post-filtering of the predicted variants. You can use 529 VarCap for single organism as well as multi organism experiments as long as you provide fasta 530 references for the involved organisms (in multifasta format). In order to allow a broad community 531 to use VarCap, we implemented VarCap within our Galaxy Server, which is publicly available at 532 http://galaxy.csb.univie.ac.at. VarCap includes default parameter settings, derived from our 533 evaluation experiments, to keep it for the user as simple as possible. The estimated runtimes for $5342 \times 0.5 / 1 / 2 \mathrm{~Gb}$ sized samples are around 35/70/150 minutes (Suppl. Figure 3 ) on an 8 core/32Gb 535 RAM virtual machine. The output of VarCap is a vcf file with a detailed description of the variants 
536 and two pdf files, which give a graphical overview of variant coverage and their frequency

537 distribution. VarCap is designed to predict different allele frequencies in experimental evolution

538 experiments, and it is able to detect and report the frequencies of multiple genotypes within clinical

539 samples e.g. multiple infections.

540 REFERENCES

Afgan E., Baker D., van den Beek M., Blankenberg D., Bouvier D., Čech M., Chilton J., Clements D., Coraor N., Eberhard C., Grüning B., Guerler A., Hillman-Jackson J., Von Kuster G., Rasche E., Soranzo N., Turaga N., Taylor J., Nekrutenko A., Goecks J. 2016. The Galaxy platform for accessible, reproducible and collaborative biomedical analyses: 2016 update. Nucleic Acids Research:gkw343. DOI: 10.1093/nar/gkw343.

Barrick JE., Colburn G., Deatherage DE., Traverse CC., Strand MD., Borges JJ., Knoester DB., Reba A., Meyer AG. 2014. Identifying structural variation in haploid microbial genomes from short-read resequencing data using breseq. BMC genomics 15:1039. DOI: 10.1186/1471-2164-15-1039.

Barrick JE., Lenski RE. 2013. Genome dynamics during experimental evolution. Nature reviews. Genetics 14:827-839. DOI: 10.1038/nrg3564.

Blount ZD., Barrick JE., Davidson CJ., Lenski RE. 2012. Genomic analysis of a key innovation in an experimental Escherichia coli population. Nature 489:513-518. DOI: $10.1038 /$ nature11514.

Bolger AM., Lohse M., Usadel B. 2014. Trimmomatic: a flexible trimmer for Illumina sequence data. Bioinformatics (Oxford, England) 30:2114-2120. DOI: 10.1093/bioinformatics/btu170.

Bos KI., Schuenemann VJ., Golding GB., Burbano HA., Waglechner N., Coombes BK., McPhee JB., DeWitte SN., Meyer M., Schmedes S., Wood J., Earn DJD., Herring DA., Bauer P., Poinar HN., Krause J. 2011. A draft genome of Yersinia pestis from victims of the Black Death. Nature 478:506-510. DOI: 


\subsection{8/nature10549.}

Chen K., Wallis JW., McLellan MD., Larson DE., Kalicki JM., Pohl CS., McGrath SD., Wendl MC., Zhang Q., Locke DP., Shi X., Fulton RS., Ley TJ., Wilson RK., Ding L., Mardis ER. 2009a. BreakDancer: an algorithm for high-resolution mapping of genomic structural variation. Nature Methods 6:677-681. DOI:

10.1038/nmeth.1363.

Chen J-Q., Wu Y., Yang H., Bergelson J., Kreitman M., Tian D. 2009b. Variation in the Ratio of Nucleotide Substitution and Indel Rates across Genomes in Mammals and Bacteria. Molecular Biology and Evolution 26:1523-1531. DOI: 10.1093/molbev/msp063.

Cingolani P., Platts A., Wang LL., Coon M., Nguyen T., Wang L., Land SJ., Lu X., Ruden DM. 2012. A program for annotating and predicting the effects of single nucleotide polymorphisms, SnpEff: SNPs in the genome of Drosophila melanogaster strain w1118; iso-2; iso-3. Fly 6:80-92. DOI: 10.4161/fly.19695.

Dalquen DA., Anisimova M., Gonnet GH., Dessimoz C. 2012. ALF-A Simulation Framework for Genome Evolution. Molecular Biology and Evolution 29:11151123. DOI: $10.1093 / \mathrm{molbev} / \mathrm{ms} 268$.

DePristo MA., Banks E., Poplin R., Garimella KV., Maguire JR., Hartl C., Philippakis AA., del Angel G., Rivas MA., Hanna M., McKenna A., Fennell TJ., Kernytsky AM., Sivachenko AY., Cibulskis K., Gabriel SB., Altshuler D., Daly MJ. 2011. A framework for variation discovery and genotyping using next-generation DNA sequencing data. Nature Genetics 43:491-498. DOI: 10.1038/ng.806.

Earl D., Bradnam K., St. John J., Darling A., Lin D., Fass J., Yu HOK., Buffalo V., Zerbino DR., Diekhans M., Nguyen N., Ariyaratne PN., Sung W-K., Ning Z., Haimel M., Simpson JT., Fonseca NA., Birol I., Docking TR., Ho IY., Rokhsar DS., Chikhi R., Lavenier D., Chapuis G., Naquin D., Maillet N., Schatz MC., Kelley DR., Phillippy AM., Koren S., Yang S-P., Wu W., Chou W-C., Srivastava 
A., Shaw TI., Ruby JG., Skewes-Cox P., Betegon M., Dimon MT., Solovyev V., Seledtsov I., Kosarev P., Vorobyev D., Ramirez-Gonzalez R., Leggett R., MacLean D., Xia F., Luo R., Li Z., Xie Y., Liu B., Gnerre S., MacCallum I., Przybylski D., Ribeiro FJ., Yin S., Sharpe T., Hall G., Kersey PJ., Durbin R., Jackman SD., Chapman JA., Huang X., DeRisi JL., Caccamo M., Li Y., Jaffe DB., Green RE., Haussler D., Korf I., Paten B. 2011. Assemblathon 1: A competitive assessment of de novo short read assembly methods. Genome Research 21:2224-2241. DOI: 10.1101/gr.126599.111.

Eyre DW., Cule ML., Griffiths D., Crook DW., Peto TEA., Walker AS., Wilson DJ. 2013. Detection of Mixed Infection from Bacterial Whole Genome Sequence Data Allows Assessment of Its Role in Clostridium difficile Transmission. PLOS Comput Biol 9:e1003059. DOI: 10.1371/journal.pcbi.1003059.

Gardy JL., Johnston JC., Sui SJH., Cook VJ., Shah L., Brodkin E., Rempel S., Moore R., Zhao Y., Holt R., Varhol R., Birol I., Lem M., Sharma MK., Elwood K., Jones SJM., Brinkman FSL., Brunham RC., Tang P. 2011. Whole-Genome Sequencing and Social-Network Analysis of a Tuberculosis Outbreak. New England Journal of Medicine 364:730-739. DOI: 10.1056/NEJMoa1003176.

Garrison E., Marth G. 2012. Haplotype-based variant detection from short-read sequencing. arXiv:1207.3907 [q-bio].

Horn M., Collingro A., Schmitz-Esser S., Beier CL., Purkhold U., Fartmann B., Brandt P., Nyakatura GJ., Droege M., Frishman D., Rattei T., Mewes H-W., Wagner M. 2004. Illuminating the evolutionary history of chlamydiae. Science (New York, N.Y.) 304:728-730. DOI: 10.1126/science.1096330.

Hu X., Yuan J., Shi Y., Lu J., Liu B., Li Z., Chen Y., Mu D., Zhang H., Li N., Yue Z., Bai F., Li H., Fan W. 2012. pIRS: Profile-based Illumina pair-end reads simulator. Bioinformatics (Oxford, England) 28:1533-1535. DOI:

10.1093/bioinformatics/bts187. 
Iqbal Z., Caccamo M., Turner I., Flicek P., McVean G. 2012. De novo assembly and genotyping of variants using colored de Bruijn graphs. Nature genetics 44:226232. DOI: $10.1038 / \mathrm{ng} \cdot 1028$.

Khan Al., Dinh DM., Schneider D., Lenski RE., Cooper TF. 2011. Negative Epistasis Between Beneficial Mutations in an Evolving Bacterial Population. Science 332:1193-1196. DOI: 10.1126/science.1203801.

Koboldt DC., Zhang Q., Larson DE., Shen D., McLellan MD., Lin L., Miller CA., Mardis ER., Ding L., Wilson RK. 2012. VarScan 2: somatic mutation and copy number alteration discovery in cancer by exome sequencing. Genome research 22:568576. DOI: $10.1101 / \mathrm{gr} .129684 .111$.

Kofler R., Langmüller AM., Nouhaud P., Otte KA., Schlötterer C. 2016. Suitability of Different Mapping Algorithms for Genome-wide Polymorphism Scans with PoolSeq Data. G3: Genes|Genomes|Genetics:g3.116.034488. DOI: 10.1534/g3.116.034488.

Kofler R., Schlötterer C. 2014. A Guide for the Design of Evolve and Resequencing Studies. Molecular Biology and Evolution 31:474-483. DOI: 10.1093/molbev/mst221.

Koonin EV., Wolf YI. 2008. Genomics of bacteria and archaea: the emerging dynamic view of the prokaryotic world. Nucleic Acids Research 36:6688-6719. DOI: 10.1093/nar/gkn668.

Köser CU., Holden MTG., Ellington MJ., Cartwright EJP., Brown NM., Ogilvy-Stuart AL., Hsu LY., Chewapreecha C., Croucher NJ., Harris SR., Sanders M., Enright MC., Dougan G., Bentley SD., Parkhill J., Fraser LJ., Betley JR., Schulz-Trieglaff OB., Smith GP., Peacock SJ. 2012. Rapid Whole-Genome Sequencing for Investigation of a Neonatal MRSA Outbreak. New England Journal of Medicine 366:2267-2275. DOI: 10.1056/NEJMoa1109910.

Li H. 2013. Aligning sequence reads, clone sequences and assembly contigs with BWA- 
MEM. ArXiv e-prints 1303:3997.

Li H. 2014. Toward better understanding of artifacts in variant calling from highcoverage samples. Bioinformatics 30:2843-2851. DOI:

10.1093/bioinformatics/btu356.

Li H., Durbin R. 2009. Fast and accurate short read alignment with Burrows-Wheeler transform. Bioinformatics (Oxford, England) 25:1754-1760. DOI:

10.1093/bioinformatics/btp324.

Li H., Handsaker B., Wysoker A., Fennell T., Ruan J., Homer N., Marth G., Abecasis G., Durbin R. 2009. The Sequence Alignment/Map format and SAMtools. Bioinformatics 25:2078-2079. DOI: 10.1093/bioinformatics/btp352.

Maharjan RP., Gaffé J., Plucain J., Schliep M., Wang L., Feng L., Tenaillon O., Ferenci T., Schneider D. 2013. A case of adaptation through a mutation in a tandem duplication during experimental evolution in Escherichia coli. BMC Genomics 14:441. DOI: 10.1186/1471-2164-14-441.

McElroy K., Thomas T., Luciani F. 2014. Deep sequencing of evolving pathogen populations: applications, errors, and bioinformatic solutions. Microbial Informatics and Experimentation 4:1. DOI: 10.1186/2042-5783-4-1.

McKenna A., Hanna M., Banks E., Sivachenko A., Cibulskis K., Kernytsky A., Garimella K., Altshuler D., Gabriel S., Daly M., DePristo MA. 2010. The Genome Analysis Toolkit: a MapReduce framework for analyzing next-generation DNA sequencing data. Genome research 20:1297-1303. DOI: 10.1101/gr.107524.110.

Minoche AE., Dohm JC., Himmelbauer H. 2011. Evaluation of genomic high-throughput sequencing data generated on Illumina HiSeq and Genome Analyzer systems. Genome Biology 12:R112. DOI: 10.1186/gb-2011-12-11-r112.

Moran NA., McLaughlin HJ., Sorek R. 2009. The Dynamics and Time Scale of Ongoing Genomic Erosion in Symbiotic Bacteria. Science 323:379-382. DOI: 10.1126/science.1167140. 
Nielsen R., Paul JS., Albrechtsen A., Song YS. 2011. Genotype and SNP calling from next-generation sequencing data. Nature Reviews Genetics 12:443-451. DOI: 10.1038/nrg2986.

Patel RK., Jain M. 2012. NGS QC Toolkit: A Toolkit for Quality Control of Next Generation Sequencing Data. PLoS ONE 7:e30619. DOI: 10.1371/journal.pone.0030619.

Pruitt KD., Tatusova T., Brown GR., Maglott DR. 2012. NCBI Reference Sequences (RefSeq): current status, new features and genome annotation policy. Nucleic Acids Research 40:D130-D135. DOI: 10.1093/nar/gkr1079.

Pulido-Tamayo S., Sánchez-Rodríguez A., Swings T., Van den Bergh B., Dubey A., Steenackers H., Michiels J., Fostier J., Marchal K. 2015. Frequency-based haplotype reconstruction from deep sequencing data of bacterial populations. Nucleic Acids Research:gkv478. DOI: 10.1093/nar/gkv478.

Qin J., Li R., Raes J., Arumugam M., Burgdorf KS., Manichanh C., Nielsen T., Pons N., Levenez F., Yamada T., Mende DR., Li J., Xu J., Li S., Li D., Cao J., Wang B., Liang H., Zheng H., Xie Y., Tap J., Lepage P., Bertalan M., Batto J-M., Hansen T., Le Paslier D., Linneberg A., Nielsen HB., Pelletier E., Renault P., SicheritzPonten T., Turner K., Zhu H., Yu C., Li S., Jian M., Zhou Y., Li Y., Zhang X., Li S., Qin N., Yang H., Wang J., Brunak S., Doré J., Guarner F., Kristiansen K., Pedersen O., Parkhill J., Weissenbach J., Antolin M., Artiguenave F., Blottiere H., Borruel N., Bruls T., Casellas F., Chervaux C., Cultrone A., Delorme C., Denariaz G., Dervyn R., Forte M., Friss C., Guchte M van de., Guedon E., Haimet F., Jamet A., Juste C., Kaci G., Kleerebezem M., Knol J., Kristensen M., Layec S., Roux KL., Leclerc M., Maguin E., Minardi RM., Oozeer R., Rescigno M., Sanchez N., Tims S., Torrejon T., Varela E., Vos W de., Winogradsky Y., Zoetendal E., Bork P., Ehrlich SD., Wang J. 2010. A human gut microbial gene catalogue established by metagenomic sequencing. Nature 464:59-65. DOI: 


\subsection{8/nature08821.}

Rausch T., Zichner T., Schlattl A., Stütz AM., Benes V., Korbel JO. 2012. DELLY: structural variant discovery by integrated paired-end and split-read analysis. Bioinformatics 28:i333-i339. DOI: 10.1093/bioinformatics/bts378.

Schmieder R., Edwards R. 2011. Quality control and preprocessing of metagenomic datasets. Bioinformatics:btr026. DOI: 10.1093/bioinformatics/btr026.

Wilm A., Aw PPK., Bertrand D., Yeo GHT., Ong SH., Wong CH., Khor CC., Petric R., Hibberd ML., Nagarajan N. 2012. LoFreq: a sequence-quality aware, ultrasensitive variant caller for uncovering cell-population heterogeneity from highthroughput sequencing datasets. Nucleic acids research 40:11189-11201. DOI: 10.1093/nar/gks918.

Yang X., Chockalingam SP., Aluru S. 2013. A survey of error-correction methods for next-generation sequencing. Briefings in Bioinformatics 14:56-66. DOI: 10.1093/bib/bbs015.

Ye K., Schulz MH., Long Q., Apweiler R., Ning Z. 2009. Pindel: a pattern growth approach to detect break points of large deletions and medium sized insertions from paired-end short reads. Bioinformatics (Oxford, England) 25:2865-2871. DOI: 10.1093/bioinformatics/btp394.

Yost SE., Alakus H., Matsui H., Schwab RB., Jepsen K., Frazer KA., Harismendy O. 2013. Mutascope: Sensitive Detection of Somatic Mutations from Deep Amplicon Sequencing. Bioinformatics:btt305. DOI: 10.1093/bioinformatics/btt305. 
544 Table 1 Detection sensitivity and precision of the combined workflow for a different number of

545 callers and at different simulated minor allele frequencies (MAF) and minimum relative

546 abundance (MRA) cutoffs. The table shows the numbers for the observed true positives (TP), false negatives (FN), false positives (FP), sensitivity and precision of the combined workflow at MRAs of 10, 5, 2 and $1 \%$ under the requirement that either 1 or 2 callers (Min Caller) had to confirm each variant.

\begin{tabular}{|c|c|c|c|c|c|c|c|}
\hline \multirow{4}{*}{1} & 20 & 10 & 139 & 2 & 0 & 0,986 & 1 \\
\cline { 2 - 8 } & 10 & 5 & 137 & 4 & 0 & 0,972 & 1 \\
\cline { 2 - 8 } & 4 & 2 & 138 & 3 & 0 & 0,979 & 1 \\
\cline { 2 - 8 } & 4 & 1 & 141 & 0 & 1238 & 1,000 & 0,102 \\
\cline { 2 - 8 } & 20 & 10 & 135 & 6 & 0 & 0,957 & 1 \\
\cline { 2 - 8 } & 10 & 5 & 133 & 8 & 0 & 0,943 & 1 \\
\cline { 2 - 8 } & 4 & 2 & 133 & 8 & 0 & 0,943 & 1 \\
\hline
\end{tabular}

Table 2 Experimental validation of a subset of the predicted variations. Three variant positions at different frequencies were amplified by PCR, cloned and Sanger sequenced for validation.

\begin{tabular}{|l|l|l|l|l|}
\hline Position & Frequency & Clones total & Clones supportive & Sanger confirmed \\
\hline 1338568 & 28 & 16 & 6 & Yes \\
\hline 1339720 & 11 & 16 & 2 & Yes \\
\hline 1339224 & 4 & 16 & 1 & Yes \\
\hline
\end{tabular}

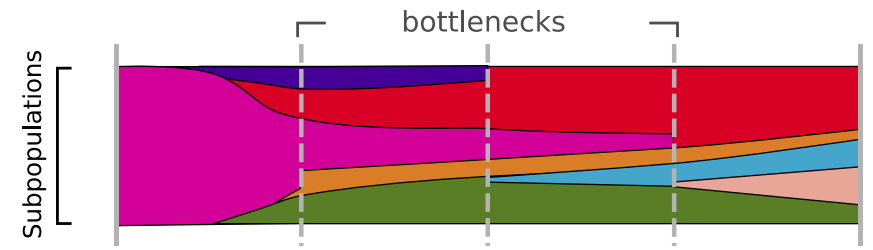

starting culture timepoint 1

timepoint $n$ generations

Fig. 1. The dynamics of a bacterial population. Alternate alleles arise over successive generations. They encounter positive or negative selection and bottlenecks, which shape the diversity landscape of a population over time. 
A

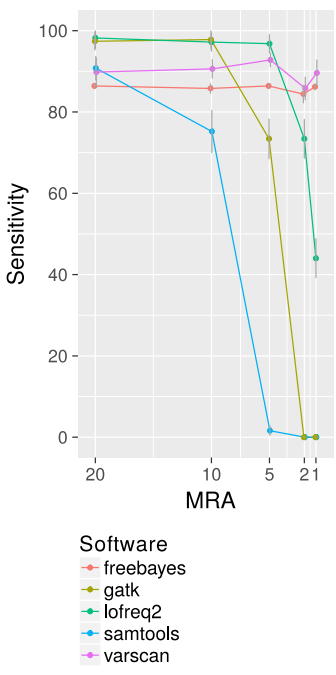

B

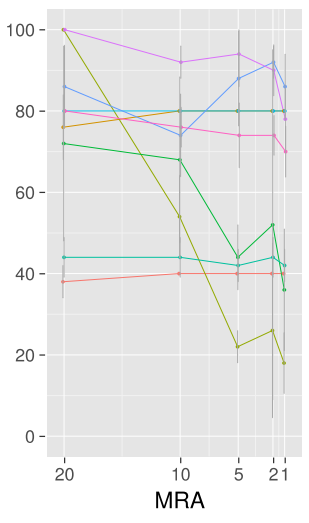

Software

- breakdancer_indel_large

- breakdancer indel large de

- cortex_indel_large

- cortex indel large in

delly_indel_large

delly_indel_large_d

- varscan indel smal
C

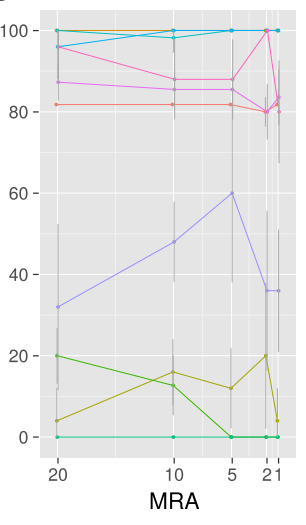

Software

- breakdancer sv

- breakdancer_sv_del

- cortex_inv

- cortex_sv

- delly_inv

- delly_sv

- delly_sv_del

- pindel_inv

- pindel_sv del

Figure 2 Analysis of the detection rate of variants with regard to Minimum Relative

561

562

563

564

565

566

A

568

569

570

571

572

573

574 Abundance (MRA), variant type and different variant calling software. It shows the detection rate of different SNP (Fig. 2A), InDels (small denotes small InDel, Fig. 2B) and SV callers (Fig. 2C) with respect to the MRA frequencies of 20, 10, 5, 2 and $1 \%$. For breakdancer, pindel, delly and cortex, two values are given: detection rate of all InDels and specific detection rate for deletion or insertion only.
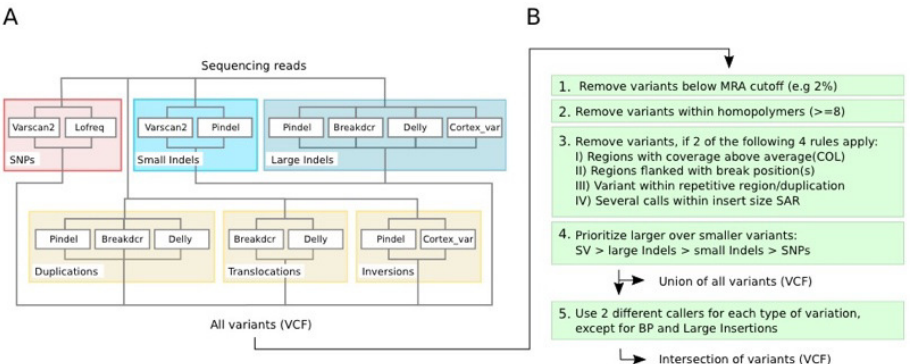

Figure 3 Detection capabilities of different tools at low frequencies. Fig. 3A shows the variant types that were successfully detected by the different software tools while Fig. 3B shows the post filtering steps to eliminate false positives. The post filtering step generates 2 output files: One file includes the union of all variants, while the other contains the intersection of variants (except break positions (BP) and large insertions, which are also reported as single calls). 


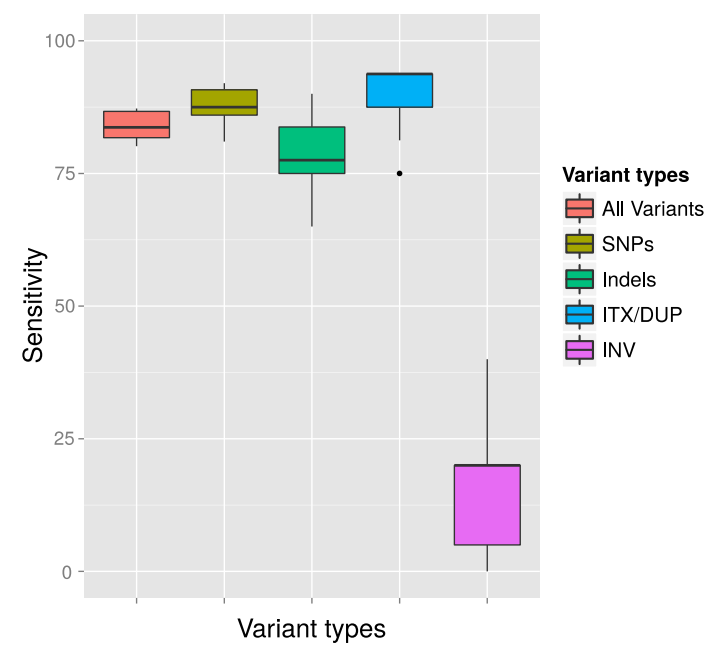

576 Figure 4 Average detection rates and standard deviation of all callers for variants simulated at a 577 MAF of $4 \%$ and a MRA cutoff of $2 \%$. The variant detection rates are shown in percent for all 578 variants(ALL), only SNPs(SNP), only InDels (IND), duplications and translocations (ITX/DUP) 579 and inversions (INV). The results show the expected sensitivity of VarCap, as we use a MRA of $5802 \%$ as a default setting to avoid false positives.

581

\begin{tabular}{|c|c|c|c|c|c|c|}
\hline & \multicolumn{5}{|c|}{ COVERAGE } \\
\hline & & 80 & 160 & 400 & 800 & 1600 \\
\hline & 4 & 0 & 4.83 & 115.42 & 1962.25 & 935.17 \\
\hline & 6 & 0 & 0 & 0.58 & 25.50 & 934.83 \\
\hline & 8 & 0 & 0 & 0 & 0.33 & 30.33 \\
\hline MAA & 10 & 0 & 0 & 0 & 0 & 1.50 \\
\hline & 12 & 0 & 0 & 0 & 0 & 0 \\
\hline & 16 & 0 & 0 & 0 & 0 & 0 \\
\hline & 20 & 0 & 0 & 0 & 0 & 0 \\
\hline
\end{tabular}

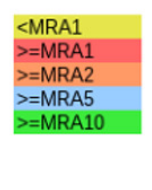

Figure 5 Influence of total coverage and MAA on FP rate. The table numbers show the FP per Megabase in context to coverage and MAA while the different colors indicate the corresponding MRA levels. We simulated MAFs of 20,10, 4, 2 and 1\% (using the sim_135VAR dataset) and detected at MAA cutoffs from 4 to 20 bases to support a variant. 
A

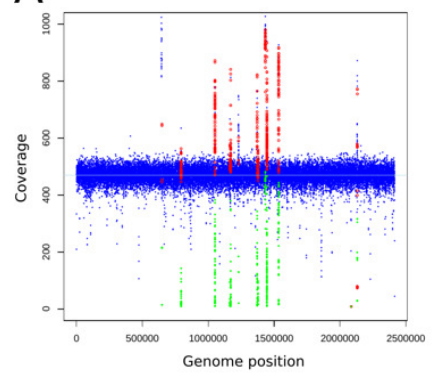

C

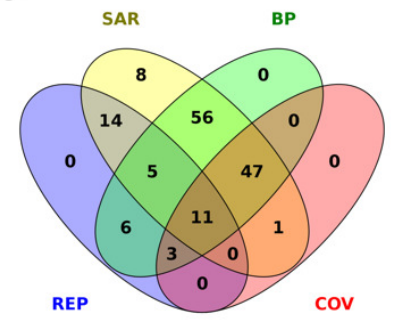

B

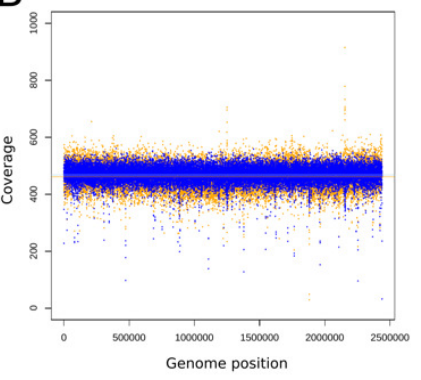

Figure 6 Coverage plots of simulated and re-sequenced data. The simulated reads without variants were mapped back to an incomplete reference (6A) and the complete reference (6B). The blue circles denote the total coverage along the genome while the green diamonds show the coverage of the FP variants and the red circles the total coverage at the FP positions. As a comparison, we show the coverage distribution of sequenced reads against the complete reference in orange in the background of $6 \mathrm{~B}$. The coverage peaks at 1220000 and 2150000 are due to additionally mapped mitochondrial reads. The light blue and orange lines show the average coverage distribution along the genome. 149 of 154 of the FP from 6A could be tagged and filtered by the properties coverage (COV), within repetitive region (REP), within SNP accumulating region (SAR) and located close to a break position (BP) as shown in 6C, the 600 remaining 5 were single calls and thus eliminated by the constraint of 2 callers per variant. 

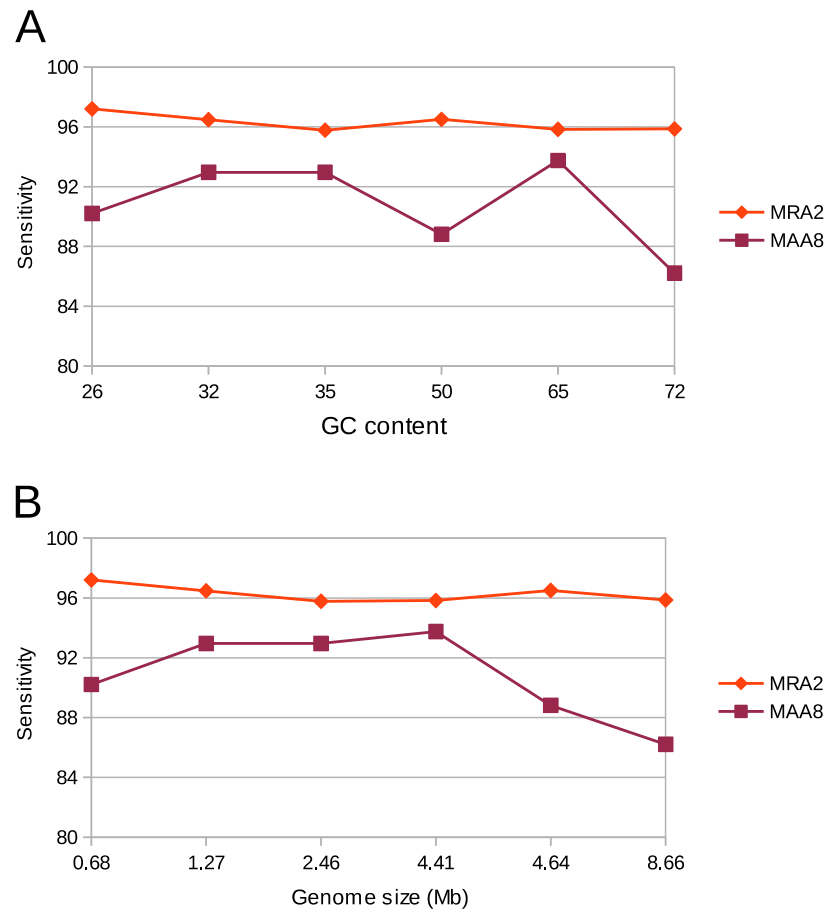

601

Figure 7 Detection rate of variants in various genomes at minimum absolute and relative abundance. The observed percentage of True Positives is shown for six organisms with differing GC content (7A) and genome size (7B). The total coverage is at 400x, the coverage of the subpopulation containing 135 variants is at 16x. No False Positives were observed at the MAA of 8 and MRA of $2 \%$.

\section{A}

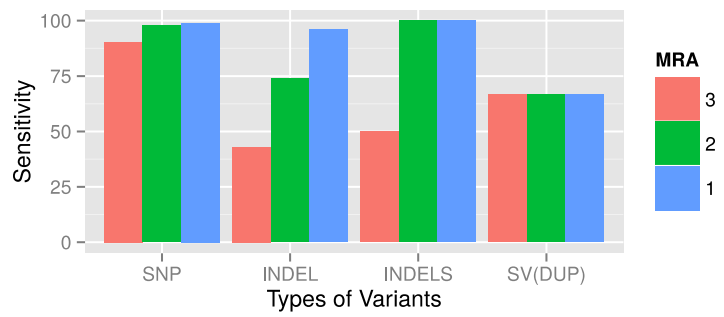

B

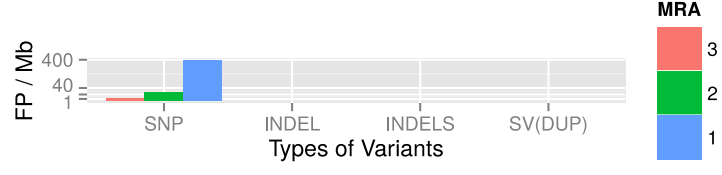

C

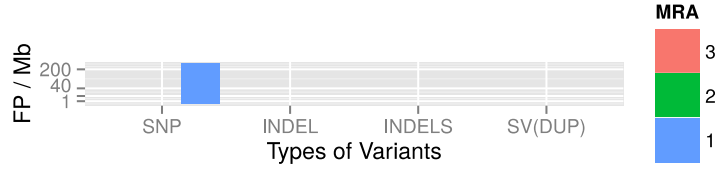

Figure 8 Observed detection rates of variants which were simulated using a genome evolution software (ALFSim) and detected at different minimum abundances. Simulated variants included SNPs, small InDels (INDELS), large InDels (INDEL) and structural variations (SV) as 
613 Positives for SNPs as counts per Magabase at the different MRAs. At these minimum

614 abundances, no FP for InDels and SV were detected. 8C shows the FP per Megabase after 615 filters have been applied.

616

617

618

619

620

621

$\begin{array}{ll}\text { SNP } & \text { Single nucleotide polymorphism } \\ \text { INDEL } & \text { Large InDels } \\ \text { INDELS } & \text { Small Indels (<10nt) } \\ \text { SV(DUP) } & \text { Duplication }\end{array}$

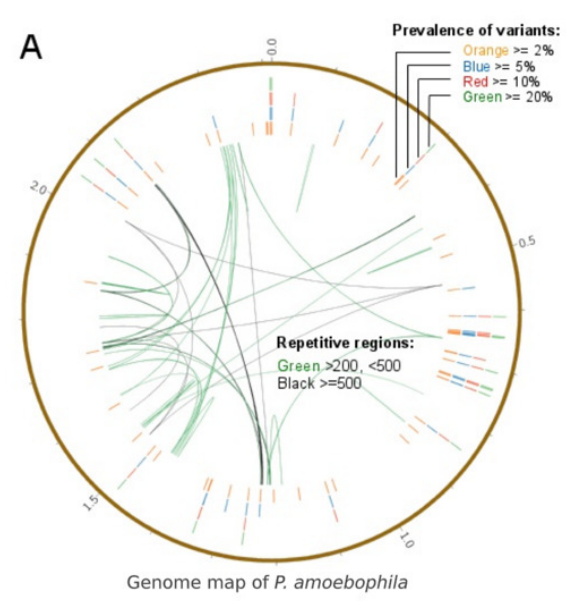

\begin{tabular}{l|cccc} 
B & MRA2 & MRA5 & MRA 10 & MRA 20 \\
\hline SNP & 34 & 23 & 16 & 11 \\
Indel & 20 & 8 & 8 & 5 \\
SV & 17 & 9 & 8 & 8 \\
& \multicolumn{4}{c}{ Variant counts }
\end{tabular}

623 Figure 9 Prevalence of variants within a long-term culture with respect to their MRAs. 9A shows

624 the prevalence of variations at MRAs of $20 \%, 10 \%, 5 \%$ or $2 \%$, which are visible in the four 625 differently colored outer circles and the presence of repetitive regions within the reference 626 genome (inner connective lines). 9B shows a more detailed view of the number of variations 627 found at MRAs of $20 \%, 10 \%, 5 \%$ and $2 \%$. 